\title{
APPLICATIONS OF ONTOLOGIES IN KNOWLEDGE MANAGEMENT SYSTEMS
}

\author{
ZOBIA REHMAN \\ PhD. Student, Faculty of Engineering and Management, Lucian Blaga University of Sibiu, Sibiu, Romania, \\ zobia.rehman@gmail.com \\ CLAUDIU V. KIFOR \\ Professor, Faculty of Engineering and Management, Lucian Blaga University of Sibiu, Sibiu, Romania, \\ claudiu.kifor@ulbsibiu.ro
}

\begin{abstract}
Enterprises are realizing that their core asset in 21st century is knowledge. In an organization knowledge resides in databases, knowledge bases, filing cabinets and peoples' head. Organizational knowledge is distributed in nature and its poor management causes repetition of activities across the enterprise. To get true benefits from this asset, it is important for an organization to "know what they know". That's why many organizations are investing a lot in managing their knowledge. Artificial intelligence techniques have a huge contribution in organizational knowledge management. In this article we are reviewing the applications of ontologies in knowledge management realm.
\end{abstract}

Key words: Ontology, Knowledge management, ontology based knowledge management.

\section{Introduction}

Knowledge management (KM) research deals with the identification, creation, representation, distribution and adaptation of heuristics of individuals or organizations. Knowledge gathering (collection of knowledge on subject matter), Knowledge organization and structuring (designing a structure for acquired knowledge for its effective management), knowledge refinement (upgraded knowledge maintenance by concerned persons) and knowledge distribution (making the knowledge available for its users) are core activities in organizational knowledge management (Benjamins, 1998). Effective knowledge management is fruitful for an organization in a number of ways; it influences core business competencies, accelerates novelty and time-to-market, strengthens decision making and organizational commitment; and helps in retaining the valuable organizational knowledge even in the absence of concerned experts (Davinport, 1998). According to (Hoven, 2001) flourishing KM is an integrated approach that combines the information that is preserved in information system of an organization with the unstructured information that lies as experience or heuristics of its people. Nowadays knowledge is encoded in electronic documents. Although electronic media allows us to create the huge volumes of documents but does not help in rapid inference through it. For its fruitful exploitation computer based tools are needed to search through files, databases and websites to extract information, capture its meaning, organize it and disseminate it (Jurisica, 1999). Such tools are more precisely known as knowledge management systems or computer based information systems and they can fall in one or more of the following groups: groupware, document management systems, expert systems, semantic networks, relational and object oriented databases, simulation tools and artificial intelligence (Gupta, 2004). In this article we will focus on how ontologies support the representation, organization, acquisition, retention and analysis of knowledge.

\section{Ontology}

Ontology is the branch of philosophy and it deals with the study of existence. In Information Science (IS), ontology is a formal representation of the knowledge of a specific domain as a set of concepts within a domain, and the relationships between those concepts. It provides shared vocabulary to model a domain and supports reasoning about concepts (Gruber, 1993). An ontology describes a domain 
using notions of concepts, instances, relations, functions and axioms. Taxonomies are used to organize the concepts in ontology and to provide the inheritance mechanism (Benjamins, 1998). Formal ontologies are in practice since $18^{\text {th }}$ century. From KM perspective, formalization of ontologies and algorithmic support to generate inferences from a given set of facts is beneficial to manage large amounts of knowledge (Jurisica, 1999).

\section{Ontologies For Knowledge Management}

Information and knowledge are the core assets of organizations in this era. Knowledge management shoulders the responsibility to manage and get benefits from these assets. That's why organizations have been investing a lot in this dimension. Ontologies have been proved to be really helpful for KM as they are applicable for information retrieval, information systems, can be used as system models, and are better way to store and retrieve knowledge semantically (Almeida, 2009). Ontology based knowledge management systems are distributed and intelligent. Ontologies constructed for information sharing and reuse are based on a predefined specification e.g. use of specified vocabulary, entities and their relationships. According to the authors in (Jurisica, 1999) artificial intelligence has brought into practice a number of ontologies and techniques to analyze knowledge represented by these ontologies. According to them, there are four broad ontological categories to deal with static, dynamic, intentional and social aspects of the world. These four categories can be used to represent knowledge of various applications. Static ontology represents existing things, their attributes and relationships. Dynamic ontology represents states, state transitions and processes. Intentional ontology specifies agents, believes and actions of agents, and social ontology depicts social phenomena of an organization. (Lehner, 2000) introduced ontologies as computer-based systems known as Organizational Memories. These systems can be used to collect and organize information systematically from different sources, in order to create a repository of organizational knowledge. In these systems the role of ontology is to deal with the syntax and semantic inconsistencies so that agents can communicate between the instances involved in a process. In the following sections the use of ontologies is explained in different phases of knowledge management.

\subsection{Knowledge Acquisition}

The Knowledge Acquisition (KA) involves the collection, analysis, structuring and validation of knowledge for representation purposes (Hua, 2008). While constructing an ontology for any domain, tacit and explicit both kinds of knowledge are needed. Explicit knowledge can be gathered from computer based documents where as for the acquisition of tacit knowledge, experts of a domain can be interviewed. The authors in (Mendonça, 2012) presented a list of steps for KA in hematology domain. To extract the domain knowledge from text documents natural language processing techniques can be applied. This task can be accomplished after the construction of a corpus for the subject domain, codification of the corpus and retrieval of information from the corpus. The second step is to interview the experts of the domain and understand their rationale to recognize the phenomena in a domain.

\subsection{Knowledge Organization And Structuring}

Author in (Poli, 1996) believes that an ontology is the general framework within which catalogues, taxonomies, and terminologies may be given an appropriate organization. In (Kumazawa, 2009) authors developed a knowledge structuring tool based on ontology. They designed a meta database, constructed a trial ontology and extracted for it concepts and relationship between those concepts from metadata. This model provided divergent exploration by tracing multi-perspective conceptual chains, contextualized the conceptual chains into multiple convergent chains, and provided help for an explorer to understand or identify an essential problem and gather existing knowledge for its solution.

\subsection{Knowledge Refinement}

The main tasks of this phase are knowledge elicitation and formalization. It is a distributive task performed by each worker. Knowledge represented in ontologies can be updated at instance level and on ontology level using annotated html pages and different ontology based tools designed for knowledge refinement (Almeida, 2009). Knowledge can also be refined by using the opinions of the experts who query the ontology of a specific domain to validate its knowledge.

\subsection{Knowledge Distribution}

Ontologies help in distributing knowledge intelligently. Web crawlers can be used to retrieve the knowledge managed in ontologies. As ontologies deal with annotated documents that's why while retrieving information from ontologies a user gets only the concerned information rather than a pool of overwhelmed documents which can or cannot contain required data. 


\section{Conclusions}

Knowledge management deals with the problem of organizational knowledge retention so that the knowledge in an organization that is acquired from experience may be reused by preserving and sharing it in a formal way. The authors in (Abecker, 2002) categorize the KM activities with respect to two aspects: the first aspect deals with the knowledge about a product registered in documents, its storage and reuse; the second aspect describes KM as a social communication process. These two aspects of knowledge management make the ontologies most suitable for KM. As an ontology is a general framework within which catalogues, taxonomies, and terminologies may be given suitable organization (Poli, 1996). Ontologies are being used in different domains of the world for knowledge gathering, structuring, refinement, and distribution and KM systems based on ontologies have been fruitful for organizations to get benefits from their knowledge.

In future we are aimed to develop an ontology based knowledge management system for preserving, sharing, and reuse the process Failure Mode and Effects Analysis knowledge generated in automotive industry. FMEA is a systematic way to identify probable failure modes and their overall effects on a system, if this analysis is timely completed, it helps to reduce the probability of risk occurrence. After FMEA valuable knowledge is produced but due to its representation in natural language this knowledge is difficult to share and reuse. We believe that ontology based knowledge preservation is most suitable solution for this problem, as ontologies can better deal with semantic representation of knowledge.

\section{References}

- Abecker, A.; Elst, L. (2002) Ontologies for information management: Balancing formality, stability, and sharing scope, Expert Systems with Applications, 23(4), p. 357-366.

- Almeida, M.B.; Barbosa, R.R. (2009) Ontologies in Knowledge Management Support: A Case Study, Journal of the American Society for Information Science and Technology, 9999, p. 1-16.

- Benjamins, V.R.; Fenzel, D.; Perez, A.G. (1998) Knowledge management through ontologies, Second Internation Conference on Practical Aspects of Knowledge Management, Basel, Switzerland, p. 5.1-5.12.

- Davinport, T.H.; Prusak, L. (1998) Working knowledge: How organizations manage what they know, Boston: Harvard Business School Press.

- Gruber, T. R. (1993) A translation approach to portable ontology specifications, Knowledge Acquisition, 5 (2), p. 199-220.

- Gupta, J.; Sharma, S. (2004) Creating Knowledge Based Organizations. Boston: Idea Group Publishing. ISBN 1-59140-163-1.

- Hoven, J. (2001) Information resources management: Foundations for knowledge management, Information Systems Management, 18(2), p. 80-83.

- Hua, J. (2008) Study on Knowledge Acquisition Techniques, 2nd Inter. Symp. On Intelligent Information Technology App.

- Jurisica, I.; Mylopoulos, J., Yu, E. (1999) Using Ontologies for Knowledge Management:An Information Systems Perspective, In Proceedings of the 62nd Annual Meeting of the American Society for Information Science, p.482-496.

- Kumazawa,T.; Saito, O.; Kozaki, K.; Matsui, T.; Mizoguchi, R. (2009) Knowledge Structuring Tool for Sustainability Science Based on Ontology Engineering, Integrated Research System for Sustainability Science and Springer, P. 1138-1143.

- Lehner, F.; Maier, R.K. (2000) How can organizational memory theories contribute to organizational memory systems? Information Systems Frontiers, 2(3/4), p. 277-298.

- Mendonça, M.;Coelho, K.; Andrade, A.Q.; Almeida, M.B. (2012)Knowledge Acquisition in the construction of ontologies: a casestudy in the domain of hematology, CEUR Workshop Proceedings, CEUR-WS.org.

- Poli, R. (1996) Ontology for Knowledge Organization, In R. Green (ed.), Knowledge organization and change, Indeks, Frankfurt, p. 313-319. 